\title{
Imposex in Thais gradata as a biomarker for TBT contamination on the southern coast of Peninsular Malaysia
}

\begin{abstract}
Thais gradata samples were collected from six locations along the southern shores of Peninsular Malaysia and analyzed for imposex incidence and organotin (butyltins (BTs), including tributyltin, dibutyltin, and monobutyltin, and phenyltins (PTs), including triphenyltin, diphenyltin, and monophenyltin concentrations), in tissue burden. On average, the BT levels were found to be higher than the levels reported a year before for other biota. In contrast, the PT compounds were found to be lower than in the previous report. The present study also classified the morphological expressions of the imposex scheme for this species into seven stages (stage 0 to stage 6) by observing the development of vas deference sequence (VDS) and penis bulk. This latest imposex scheme clearly indicates the mechanism of VDS growth, the structural changes from penis bulk to a penis with flagellum, and from a normal vaginal opening to a swollen vaginal opening. The degree of imposex was assessed using the vas deference sequence index and the percentage of females possessing the imposex characteristics. It was found that locations possessing high imposex levels also tended to show high BT levels in the snail tissue samples. However, correlation analysis did not show a significant relationship among the two parameters. A better result could be obtained if more samples and sampling locations were added in order to prove the hypothesis. The nonsignificant correlation between the shell height and any of the organotin compounds, along with no significant differences between BT levels in female and lower imposex stage samples, suggests that the BT concentration detected was a recent contamination.
\end{abstract}

Keyword: T. gradata; Imposex; Biomarker; Pollution effects; Organotins 